\title{
The Strength of Olistic Design for Organisation, between effectiveness and disruption
}

Conference Paper · December 2013

DOI: 10.1109/TIDMS.2013.6981216

CITATIONS

0

3 authors, including:

\section{Francesco Galli}

Politecnico di Milano

17 PUBLICATIONS 2 CITATIONS

SEE PROFILE
READS

11

Some of the authors of this publication are also working on these related projects: 


\section{IEEE Tsinghua International Design Management Symposium}

(TIDMS 2013)

Shenzhen, China

1-2 December 2013 


\title{
The Strength of Olistic Design for Organisation, between effectiveness and disruption
}

\author{
Francesco Galli, Marco Maiocchi, Margherita Pillan \\ Department of Design - Politecnico di Milano - Milano - Italy \\ francesco.galli@polimi.it,marco.maiocchi@polimi.it,margherita.pillan@polimi.it,
}

\begin{abstract}
Design refers today to many different aspects, such as product, interior, communication, brand, service and so on. It is possible to provide a coherent perception of a whole environment, designing all the aspects so that they can communicate coherent values and univocal interpretation. This is possible by making the design driven by principles starting from metaphoric models of the mission of a company, translating them into perceptual aspect involving both static elements (layouts, interior design, logo, etc.) and dynamic (interaction protocols, services processes, processes, and so on). Beside the evident advantages of such an approach, problems can arise from conflictual points of view between the company (intended as a unique organism with specific mission and values) and the employees (requiring personal satisfaction, not necessarily coherent with the mission of the organization).
\end{abstract}

The paper describes real experiences exemplifying what indicated, and presents: i) a quick description of the design approach able to design coherent solutions for different artifacts/ services (values identification, required emotions specification, metaphors supporting them, perceptual aspects supporting the metaphors, design of any aspect); ii) the description of a similar approach followed in designing some department in a relevant hospital; iii) the positive effect of the approach evaluated by the positive reactions of patients and nurse; iv) the opposition of the doctors, feeling themselves as interpreted less relevant for the therapies effectiveness; $v$ ) the corrective actions taken in order to avoid a disruptive effect of the cohesion between doctors (as more relevant actors of the services) and the rest of the environment

The paper describes the experience, and points out the differences between the design of artifacts for external customers (e.g., cars, white goods, etc,) and the design of environments in which customers and "producers" share the same space and processes, and suggests, for these cases, approaches going beyond User Centered Design.

A critical discussion concludes the paper.

Keywords- emotional design; olistic design; design management; design leadership,

\section{INTRODUCTION}

Design is today an activity embracing any production field. It spans from the development of many different physical artifacts (simple as glasses or chairs as well as complex as cars) to service structures and behaviors; it is coping with many fields, from industrial production to communication, to health care services, to urban furniture and so on. It is quite clear that in any field and for any kind of development, design is able to increase the value of the results by joining emotional meanings to the proper functions and usability [1] [2], and that, after the availability of industrial production methods, design is the only way for competition [3].

Despite the methods for designing simple objects or complex structures refer to the same principles, it is quite different to design a vacuum cleaner or to design a bank counter service: the main difference between the two is not the complexity, the dependency on processes or the dialogic nature of the bank interaction, but consists in a different approach toward the customer.

In fact, the User Centered Design approach, as the one sketched in Fig. 1, points on the user needs, and interprets the organizational needs as the need of the organization in which the customer is involved in using the designed artifact.

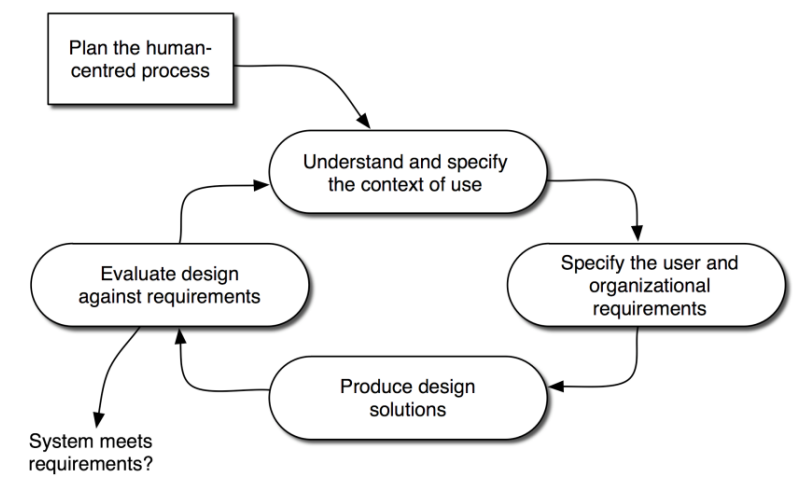

Fig.1 - A typical User Centered Design process

This approach should be improved for services in which the service provider shares environment and processes with the customers.

After a description of the followed emotional design approach, in the following we will show some examples in which lacks in the provider needs care resulted into disrupting effects in the whole organization, and proposals to overcome such a kind of problems.

\section{EMOTIONAL DESIGN}

Emotional Design in usually presented through intuitive concepts and examples, while today we are able to describe more scientifically what an emotion is and how to raise it through perception. In this chapter we present quickly an original model of Emotional design, what we mean as emotion, which perceptions can be used for raising emotions and how to drive them through metaphors.

\section{A. A model for Emotional Design}

Consider Fig. 2: when a user sees / uses/ enter in touch with an artefact / a communication message / a service, he/she is supposed to get the proper results, and in an easy way, but getting in addition meanings and emotions. 


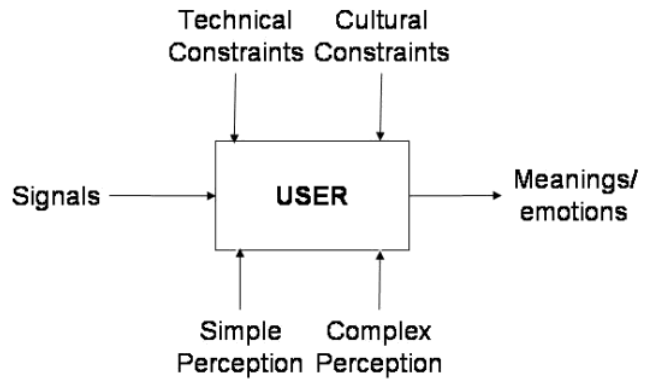

Fig. 2 - A model for Emotional Design

Signals are constituted by any kind of possible stimulus catched through our senses, on any possible transmission channel.

The emotions are driven by simple perceptions (such as cold vs warm colors, edged vs curved shapes, and so on) and by complex perceptions (mainly based on stereotypical metaphors), and both kind of perceptions can be consciously designed.

Of course, the designer must take into account technical/economic constraints, but also cultural constraints take place (e.g. some cultures are in favors of owning goods, instead of using them through services [4]).

Provided that we are able to define scientifically what is an emotion and to indicate simple and complex perception mechanisms, we will be able to design artifacts oriented to raise specific emotions.

\section{B. What is an emotion}

According to the works of many neuroscientists [5], an emotion is the excitation of a specific part of our brain by specific neurotransmitter, able to change our answer to external conditions, and developed through the species evolution as a way to increase the survival competitively. For example, fear we feel in a dangerous situation making us run fast away is the result of the excitation of some part of our reptilian brain, increasing our blood pressure and heart beat, reducing our answer to pain and to tiredness (as well as the capability of our immunity system), and to any possible sense of libido.

Panksepp describes seven main emotions, all of them related to the oldest brain parts (reptilian and old mammalian brain the the Le Doux scheme [6]); very roughly:

- SEEKING: the status pushing us to look around, to understand the surrounding, to seek for something (or without, but just for knowing);

- $\quad$ FEAR: the feeling of a danger, possibly giving us the choice "fight or flight";

- RAGE: what pushes us to attack;

- LUST: sexual push;

- CARE: the maternal love imposing care to babies;

- PANIC: the other side of care, the suffering for the absence of the parents;

- PLAY: the push toward playing, as real life imitation, for a useful social relationships building.

Some of the above emotions can be considered desirable and positive, while others are for sure negative: for example
SEEKING provides pleasure, with the same mechanisms (but without negative effects) of some drugs.

There are inner interpretation mechanisms in our brain, associating some perceptions to meanings, and then to emotions; e.g. RAGE is recognizable from facial expressions, and shapes recalling those expressions are able to rise the same emotions (e.g. the aggressiveness of some sports car, as power self-identification).

\section{Perception and metaphor}

Many perceptual structured signals can provoke emotion arousal: there are many studies an the neurobiological basis of aesthetic able to show the role of edged or curved profiles, of exaggerations, of ambiguous signals, of symmetry and many others [7], but the most powerful way to raise emotions is the metaphor.

We consider a metaphor [8] as a partial mapping between the entities and their relationships for a set of elements belonging to two different semantic fields, so that we can speak on the elements of one field with the words of the other (e.g. "your words are sweet to my ears", or "I cannot digest your words").

While the basic emotions are related to the oldest part of the brain, the cortical part is able to interpret rationally the structured signals, and to provide them with meanings derived from metaphors and stereotypes, able in turn to exite the inner brain parts.

For instance, if we look to a vacuum cleaner as the Bidone Aspiratutto (see Fig. 3), the army green, the stencils, the roughness of the shape and other similar detail suggest us metaphorically army and war, and we feel ourselves powerful and full of RAGE against our enemy: the dirty.

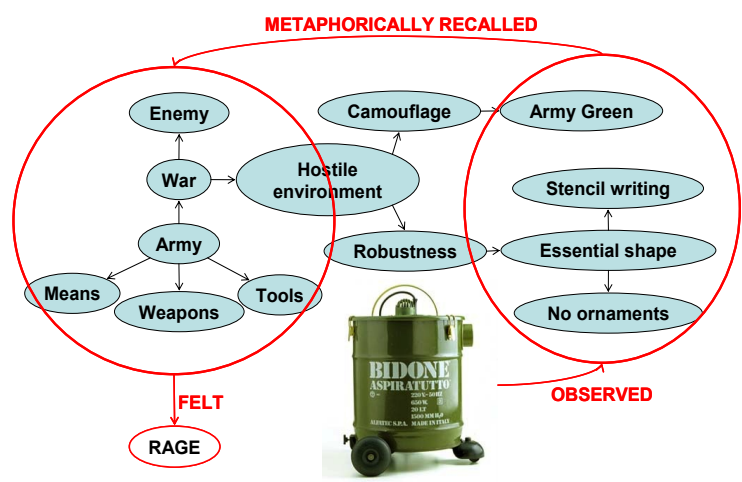

Fig.3 - Metaphoric interpretation of a vacuum cleaner

\section{Designing emotions}

Following the above model, a designer asked to design an artifact, can

- Define the usual steps as in a normal User Centred Design process (in order to guarantee the proper functions, the proper usability, the suitable costs, the proper maintenance needs, and so on);

- Define the emotion target, able to make the artefact desirable for the user;

- Provide the perceptual shapes, primary and metaphoric, able to raise the defined emotions. 


\section{AN EXPERIENCE: AN ITALIAN HOSPITAL}

While the described approach can be easily used for consumer goods, problems can be observed in applying it to wide environments, such as hospitals or other kind of organizations. In particular, we refer to a specific experience done with an Italian hospital.

\section{A. A Chance}

Following relationships between our University and an important Italian healthcare Centre, we proposed some actions, in order to change some interior aspects, not coping with medical aspects.

Our internal sponsor was the Unit of the Relationships with the Users, and, of course, it has been not possible to design actions from scratch, but just to influence the project on the perceptual aspects.

The chance came from the decision of refurbishing an entire department, in which mainly visits and examinations were performed, without any hospitalization.

\section{B. The Interventions}

We agreed with the Department Chief to change totally the interior design aspects, in order to increase three emotions arousal: SEEKING, CARE and PLAY.

The actions were constrained by the Technical Department of the hospital, imposing completely the specifications for costs and materials, and we had just the possibility to choose colors, furniture layouts, and add-on at no additional costs.

Despite those heavy constraints, the choice of unusual colors (orange walls; acid yellow, deep purple, fuchsia, brilliant green for doors, with a colour code for the kind of access; purple chairs for the waiting room, and so on), the disposition of the chairs (asymmetric rows, empty corridors, etc) was able to make the department different.

But the real change resulted from the many paintings hanged on the walls; a contest was organized, asking to many artists to provide free a work, possibly related to the activity of the department (breast radiology): the success of the initiative was astonishing, and within a couple of months we received 170 works by 160 artists from 6 countries. The paintings were very dense on the walls, and the result was surprising .

In fact SEEKING was greatly enhanced, providing the patients with a strong feeling for CARE toward them, as well as a PLAY sensation in order to discover and understand the various meanings behind.

\section{The Resulting Effect}

The operation resulted in a great success: patients changed their behaviors, expressing stress reduction, interest, positive mood changes; nurses and other technical staff expressed happiness and satisfaction; the department chief expectations was completely fulfilled.

More, many other doctors expressed their appreciation, asking for similar changes in their departments.

\section{The Recognised Problems}

Nevertheless, nothing happened after the intervention.

Despite this action was one of the many design proposals (changes in the hall, in way finding, in a communication campaign against smoking bi doctors and staff, new informative booklets, and many others), nothing followed.

According to the public declarations, everybody was positive on the changes and in the results, appreciating the approach, but none of the doctors considered the effect on the patients more than worthy of curiosity. More, some of them, required about neurosciences and psych neuroendocrinology (the clinical study of hormone fluctuations and their relationship to human behavior), revealed that "those kind of things are just similar to sorcery" and that "it is foolish to think that a color of the environment can influence a therapy". Of course, we was not supposing a direct cause-effect relationship between our project and health, but we was ready to believe a mediate affect, passing through a stress reduction, a positive more optimistic mood, and then a more constructive approach on the therapies (including a better compliance with the medical prescriptions). What we felt was a strong statement: "We are the care!"

\section{E. Other Confirmations}

Such a kind of "twofold position" in front of an action oriented to the benefits of a customer in not unusual, and could be, in our opinion, recon ducted to different personal interests in the various types of stockholders: we are not suggesting that everybody is following his/her own interest, but that the played role imposes a perception of relevance quite difficult to be accepted.

Some examples:

- During the 12th International Conference on European Integration for Surgical Know How held in Naples in the 2008, a presentation of the described approach was provided during the conference; the presentation was very appreciated by doctor's wives (as testified during the official conference dinner), but none of the doctors was minimally interested in it, devoting the time more to very expensive (and not yet working) automation in light movements in a surgery room, or to new technologies allowing virtual presence in surgery, and so on.

- In other environments, a university, despite all the professors agreed on the relevance of the management role played by many of them (dean, department chief, active participation to many consultive or executive organisms, etc.), involving heavy time spending, the majority of them decided to acknowledge those involvements with a very little extent, not comparable with the loss of time for publishing scientific papers or for holding courses: it has been clear that everybody wants rules and persons able to define the best ones, as well as to handle the consequent work, but only a minority accepted to renounce to the "scores" abilitating his/her career;

- Personal experiences of the authors revealed how also in companies well focused on a publically shared vision, key persons were oriented toward personal assertive behaviors celebrating their personality, also if in contrast with the acceptance of the shared vision;

- The analysis of the problems and the recognition of other examples in the past experiences, recalled a collection of psychological experiments carried on in many companies, conducting to similar conclusions [9].

We were then forced to consider that the followed approach, valid for a single artifact cannot be applied to large organizations: if the design is confined to a specific object, it is possible to manage it because the goal of the company (a business success) is not directly related to personal expectation of the company employees, but if design is an overall driving force, with the goal of setting up a coherent organization in developed products, issued services, organizational structure, 
architectural physical layout, and so on, conflicts can arise among the various different goals, and an User Centred Design approach can be misleading.

\section{A DifFERENT MODEL: USER CENTRED DESIGN VS COMMUNITY CENTRED DESIGN}

The done experience puts in evidence that it is quite different to design for a customer and for a community; in the former case, we can apply the traditional User Centred Design approach, while in the latter we have to cope with a larger community, sharing values as a whole, but with possible conflictual personal needs.

In broad terms, user-centered design (UCD) is a design process in which, at each stage, extensive attention is given to the needs, desires, and limitations of final users, rather than forcing the users to change their behavior to adapt themselves to the product. User-centred design foresees usually also tests of the user behavior in real world tests with real users.

A user can be initially modeled through personas, and this method has not by chance initially emphasized for software products development [10]; in fact, the complexity that can be added to a software application with marginal costs imposed to define with the best possible precision the user needs: not the possible user needs, but the specific needs of an archetypical user; the use of personas in UCD results as the identification of non conflictual features desirable by different characters of the target users.

But when we operate some design action in an hospital, our "personas" could be identified not only in different archetypical patients, but also in other role archetypes: doctors, nurse, administration staff, technical staff, managers, visitors, cleaning services, and so on.

In this case, conflicts are unavoidable: also in the most trivial activities: a bed should be designed as easy to be cleaned and to be moved, comfortable for laying and for nurse cares, located in a room assuring privacy but allowing space saving and doctor visit tours organization, standardized structures for handling purposes and pretty pieces for patients pleasure, and so on; clearly many of those aspects are strongly conflictual, and the solution cannot be to find a compromise.

This is what happened to our hospital project since start:

- the Unit of Relationship with the Users was deeply focused on the patients satisfaction;

- doctors were, generally speaking, interested in a high healing rate, possibly with scientific publishing opportunities;

- Technical Offices were interested in legal constraints, cost reduction and in on time project (is not by chance that all the architectural and furniture decisions are taken without or with a very poor interaction with doctors);

- Nurses and staff were interested in "ergonomy" for their work;

Those points of view are inherently conflictual.

We built artificial communities, in which the goals of them are "social", but not compliant with the goals of the individuals: we can observe the same in many other natural communities (such as baboons), but there are also unitary well working communities (such as ants or bees). In order to cope with the problem, we suppose to need rising up in the community cohesion.

A possible new approach for a Community Centred Design can be inspired by the European Foundation for Quality Management model [11] (Fig. 4).

ENABLERS



Fig, 4 - The EFQM model of an organization.

According to the model, an organization must simultaneously get the following results (from right to left):

- $\quad$ provide business results (with economic advantages, or social, according to the mission);

- get the above results guaranteeing the employees (people) satisfaction, in order to keep them happy and loyal;

- The same for the customer satisfaction;

- The above results must be obtained with care for the society and for the environment (advantages for all, no pollution, etc.).

Those results can be obtained through the following enablers (from left to right):

- a strong leadership, able to inspire a vision and to make it a driving force for everybody;

- the best management for the human resources;

- a strong care for the management and for the handling of any kind of needed resources (financial, raw matters, etc.);

- a transparent and shared communication on the organization policies and strategies, so that people is not simply aware on the goals, but committed on the complete organization life.

According to the model, a good management of the organization processes allows to design the organization itself and to rule it during its life, to reach the results.

The weak point of this model is the first enabler: the leadership, more and more scarce resource; the growths of multinational companies and the consequent rise of a managers class in opposition to enlightened entrepreneurs is going more and more against the availability of strong leaders with wide visions.

Further problems arise in public organizations, in which the upper management positions have a duration limit (typically three years), imposing rotations, and then difficulties in maintaining stable visions. 


\section{CRitical Conclusions: The ApProach We Should} TAKE.

If we wanted to adopt the EFQM model in our above reported experiences, , we should have carried on a different road map of the project:

- to identify as main goals the success of the organization in terms of healing success at a reasonable cost; this goal can be obtained not only through the various internal processes and the medical excellence, but, from an emotional point of view, also through a proper communication, possibly coping with a mix of RAGE (the most used metaphor in the western medicine is the war against the disease) and CARE ;

- to take into account the people satisfaction, in terms of working activities and role recognition: the employees, at any level and in any situation, should be surrounded by a physical environment, by working tools, by relationships with other colleagues and with the organization able to raise SEEKING (a principle of pleasure), PLAY and CARE (with a double effect: to feel themselves cared by the organization, by colleagues and by customers, and to inspire them CARE towards organization, colleagues and customers);

- to take into account the customer satisfaction, i.e. to make the patients experience as best as possible, reducing the stress and stimulating positive emotions, mainly SEEKING, CARE and PLAY, and fighting FEAR and PANIC; this can be well organized by modeling the patients experience as a journey through the healing process; by identifying the various steps, the patient experience is constituted by the sequence information, check-in, waiting, visit(s), waiting, exam(s), waiting, therapy, waiting, and so on, with possible frequent recycles; because each of those steps are related to specific environments and relationships, for each of them proposals can be provided for rising emotions, both through "static aspects" (furniture, layouts, ...) and "dynamic" (behaviors and dialog protocols with staff, ...) [12] [13] Asylum.];

- to consider, in any design step, sustainability environment aspects, as well as and cultural values (f.i. Italy is less sensitive to the familiar group in respect to China, and this could change logistic aspects in visiting hours, places, and so on); of course a proper communication cools stimulate SEEKING, CARE and PLAY.

In order to avoid conflicts among the above points, it is necessary that Policies and Strategies are well communicated and able to rise SEEKING and RAGE (in term of proudness for participating to the mission), and the Human Resources management should heavily support them, also by rewarding positive behaviors.

All the above aspects are scarcely able to get the expected results without a proper leadership: often great entrepreneurs were able to embody the above philosophy in an intuitive way (for instance, in the past Adriano Olivetti built a company able to live in a strong positive symbiosis with the local community in Ivrea and in Italy; in recent years Steve Jobs set up a company able to interpret excellence and exclusivity both for customers and employees).

In our case (hospitals and Italy) we have to choices: either to find the illuminated entrepreneur owning a private healthcare structure, or to find the stability point in the public healthcare centers.

We choose the second way, more difficult but wider in the action:. In the Italian regulation, healthcare is under the responsibility of twenty Regions (i.e. the first level administrative divisions of the state); Regions have the power to issue laws ruling their competences, and among them healthcare. So we provided to Regione Lombardia (the Region to which Milano belongs) a proposal of a complex set of specifications, involving all the above design aspects, and interaction protocols (also in terms of relationships between patients and doctors/nurse/staff) to be considered integral part of any new healthcare project. This proposal is non oriented to define implementation details, but to describe all the principles behind the projectual choices, asking for each of them the declaration of the rationales.

We are confident that such a proposal could be really a turning point for public healthcare, and a good experience in Community Centred Design: iif our proposal will be accepted quickly or not is another, different story.

\section{REFERENCES}

[1] T. van Gorp, E. Adams, Design for emotions, Elsevier, 2012

[2] D. Norman, Emotional design, Basic Books, 2005

[3] M. Maiocchi, M. Pillan, Design e comunicazione, Alinea, 2009

[4] G. Hofstede, G. J. Hofstede, M. Minkov, Cultures and organizations: Software of the mind. 3rd Edition, McGraw-Hill USA,2010

[5] J. Panksepp, L. Biven, Archaeology of mind:neuroevolutionary origins of human emotion, New York: W. W. Norton \& Company, 2010

[6] J. LeDoux, The emotional brain: The mysterious underpinnings of emotional life, 1996,Simon \& Schuster, 1998

[7] V. S. Ramachandran, W. Hirstein, The science of art. A neurological theory of aesthetic experience, Journal of Consciousness Studies, 6, No. 6-7, 1999, pp. 15-51

[8] G. Lakoff, M. Johnson, Metaphors we live by, University of Chicago Press, 1980

[9] M. Selvini Palazzoni, Sul fronte dell'organizzazione, Feltrinelli, Milano, 1981

[10] A. Cooper, The Inmates are Running the Asylum, Sams Publishing, 2004

[11] EFQM Excellence Model 2013, Brussels. EFQM. 2012

[12] M. Maiocchi, A. Pollo, Health and Design, Design2Business International Conference, Beijing, 2009

[13] Zhabiz Shaifeyoun, research on progress as a $\mathrm{PhD}$ project at the Politecnico di Milano, unpublished 


\section{Table of Contents}

\section{Part 1: Design Management, Innovation, and Market Exploitation}

P1-0529-02 Networks: Open, Closed or Complex-Connecting philosophy, design and innovation Jamie Brassett

P1-0530-02 Saudi-China Friendship-From relations to innovations

Bakhotmah, Fawaz Abid,Mingxi Tang

P1-0530-03 It's not about the Planet-Embracing eco-design, driving competitive advantage Richard S.G. Chipps

P1-0621-01 Team Equilibrium and Innovation Performance

Marco Vinicio Ferruzca Navarro,Josep $M^{a}$ Monguet Fierro,Jorge Rodrigues Martinez,Alejandro Trejo Omeñaca

P1-0621-02 From Production to Purpose-Using design led innovation to build strategic potential in a family-owned SME

Anja Krabye,Cara Wrigley,Judy Matthews,Sam Bucolo

P1-0624-01 Six Models of Managing Design in Manufacturing Industry

Xihui Liu

P1-0628-02 Teaching Design Thinking and Design Led Innovation to Non-Designers: a Tertiary Facilitator Multidisciplinary Study

Jonathan Morehen, Cara Wrigley, Natalie Wright

P1-0629-02 The Strength of Olistic Design for Organisation, between Effectiveness and Disruption

Francesco Galli, Marco Maiocchi, Margherita Pillan

P1-0630-05 Designing Innovative Business Models:Five emerging meta-models

Dean Johnson, Cara Wrigley, Karla Straker,Sam Bucolo

P1-0630-06 Design Management in Korean Entertainment Business Hyobin Jung, Hyoung-wook Jo, Kyung-won Chung

P1-0630-14 Discuss of Chinese Industrial Structure Adjustment Based on Design Innovation Hong Hu,Nan Gao

P1-0630-17 Exploring Photography as A Method for Research Yongsheng Wang, Hua Dong

P1-0630-21 Research on Internal and External Mechanisms of Attributes Transmutation and 96 Dissimilation during Product Evolution-The case of personal mobile communication terminal Qiwei Liang, Liqun Zhang

P1-0708-02 Design as a Transformative Force inside Business Organizations-A case study of a socio cultural center located in a marginal zone of Mexico City Cruz-Megchun, Beatriz Itzel 
P1-0709-01 A Review of Global Design Economies:Implications for the future of the profession \& for policy formation

Alex Williams

\section{Part 2. User Study, Design Thinking and Design Innovation}

P2-0517-01 Forms, Illustrations and Color in the Design of Children's Books

Hongcheng Li, Weiping Hu, Yanlei Wang

P2-0519-01 Research on Information Visualization Design Based on the Cognitive Theory

Tao Xi

P2-0523-02 Design Driven Product Innovation in Enhancing User Experience Oriented 127 Organisational Culture in B-to-B Organisations

Maarit Heikkinen, Hannamaija Määttä

P2-0527-01 Exploring Innovative Ways for Companies to Engage with Customers through the Internet in Developing New Products

Wei Liu,James Moultrie

P2-0529-01 Design Thinking as a Disruptive Discourse Embracing Conflict as a Creative Factor Francesco Galli, Irina Suteu

P2-0530-08 The Design Analysis of Products to Alleviate Human Fatigue Ying Zhong

P2-0626-03 Design as A Facilitator-Thinking of designer's role and skills as facilitator in the complex context

Churan Yin

P2-0627-02 Discovering and Developing the "Insight" by Opportunity-oriented User Research Jian Guan, Jun Cai, Jikun Liu

P2-0629-01 Exploring the Norms for the UX Design of Intelligent Products: A Case Study Chensheng Wang, Liang Chen, Lichang Zhao,Mengyan Li

P2-0630-18 A Study of Urban Youth Classification using Self-knowledge Method Honghai Li,Xin Liu,Bingxue Gao

P2-0630-22 Techniques Employed in Nudging People to Make Better Choices Xi Ai, Ying Zhang

P2-0729-01 Power of Ideas in Shaping and Delivering Design Business Success

Simon Bolton, Camille Chinneck

\section{Part 3. Brand, Design Tactic and Product management}

P3-0523-01 Brand Passionate Consumption: What is it?How does it work?

Hui Zhong, YanJie Zhang

P3-0622-01 Design Identity System Based on Product Identity and its Case Studies 
P3-0627-01 A Conceptual Designer-led New Product Development Process Model for Chinese 200 SMEs

Ke Lou, Darren John Southee, Erik Bohemia

P3-0629-05 Design Innovation-The role of design in combining process and product innovation Mingchang $W u$

P3-0717-01 Importance of Vendor and Manufacturer Relationship for Effective Lean Practices Muhammad Rafid Mahmood, Usama Bin Perwez, Anum Abdul Salam

P3-1001-02 Study on Interactive Product Experience Design Methods based on MAX6\MSP Yanrui Qu, Wen Su, Yanshan Peng

\section{Part 4. Design and Business Innovation Strategies}

P4-0522-01 Towards Creating a Sustainable Design Ecosystem for MSMEs-A Design Clinic 230 Approach Shashank Mehta

P4-0531-01 Embedding Design Capacity in Research Driven Innovation Teams Lisa Malmberg,Stefan Holmlid

P4-0618-01 The internationalization of Mexican Design:The case of design firms and individual designers

Jorge Rodríguez-Martínez,C. Sue Andrade-Díaz,Marco Vinicio Ferruzca-Navarro

P4-0628-01 Design LED Innovation: Shifting from Smart Follower to Digital Strategy Leader in 251 the Australian Airport Sector Rebecca Price,Alexander Dreiling, Cara Wrigley,Sam Bucolo

P4-0629-04 Early Challenges of Shifting an Australian Manufacturer's Utilisation of Design Rohan Doherty, Cara Wrigley,Judy Matthews,Sam Bucolo

P4-0630-04 Making Sense of Purpose, Direction and Innovation:An embedded design led innovation case study in the Australian Mining industry

Peter Townson,Judy Matthews, Cara Wrigley,Sam Bucolo

P4-0630-08 Using the Methods Designers Use as Aids to Thinking-The case of public-private partnerships in sustainable agricultural value chain development Niti Bhan,Bart Doorneweert

P4-0630-10 The Challenges of Adopting Design-Led Innovative Strategies in Not for Profits-The role of consumers, culture and employees Erez Nusem,Cara Wrigley,Judy Matthews,Sam Bucolo

P4-0630-19 Make a Stop for a Bookshop Applying design to create a holistic experience for chinese bookshop Haochen Wang

\section{Part 5. Service, System Designs and Business Model}


P5-0620-01 Matching the Innovation of Products with Organizational Change: Introducing 303 Design Knowledge into Chinese Furniture Manufacturing Companies

Xiaobo Qian,Alessandro Deserti

P5-0628-05 Can Emotion Provide a New Approach to Business Model Innovation?

Karla Straker, Cara Wrigley, Michael Rosemann

P5-0628-06 The Role of Servicescapes as Touchpoints for Different Service Settings

Kyongsill Lee, Kyung-won Chung, Ki-Young Nam

P5-0630-07 Design Led Innovation to Rejuvenate Local Food Systems and Healthy Communities:

An Emerging Research Agenda

Emily Ballantyne-Brodie, Cara Wrigley

P5-0630-11 Changing Human Behavior Through the Options and Feedback Design of Service Systems

Zhenwei You, Haruo Hibino, and Shinichi Koyama

P5-0701-01 Service Design Tools as Frameworks in the Generation of Business Ideas-An action research case study

Laura Mata García,Alessandro Deserti,Carlos Teixeira

P5-0703-01 Does Visual Communication Get Through to Its Audiences

Tore Kristensen, Gorm Gabrielsen

\section{Part 6. Intellectual Property and Design Management Education}

P6-0605-01 Dancing with Ambiguity-Design thinking in interdisciplinary engineering education

Ville Taajamaa,Heikki Sjöman,Senni Kirjavainen,Tuuli Utriainen,Lauri Repokari,Tapio Salakoski

P6-0626-02 Chinese Students' Conceptions on a Sino-Finnish Joint Program of International Design Business Management Fei Fan

P6-0628-10 Intellectual Property Protection in Design Management Liang Jing

P6-0629-06 IP, an Interesting Phenomenon-The relevance of patents for the design-led start-up business Matthias Hillner

P6-0630-13 Engaging Design Students in User Research

Hua Dong,Shu Yuan

P6-0708-03 Do Design Management Curricula Meet Current Global and Local Practice in the UAE?

Cruz-Megchun, Beatriz Itzel 